\title{
Influence of Many-Month Exposure to Light with Shifted Wave Range and Partial Fullerene Hyperpolarization on the State of Elderly Mice
}

\author{
Sergiy A. Gulyar ${ }^{1,2}$ and Zynaida A. Tamarova ${ }^{1}$ \\ 1. Department of General Physiology of Nervous System, Bogomoletz Institute of Physiology National Academy of Sciences of \\ Ukraine, Kiev 401601, Ukraine
}

2. International Medical Innovation Center Zepter, Kiev 02152, Ukraine

\begin{abstract}
Part of the unidirectional light waves (background polarization) due to the passage through the layer of fullerene molecules, acquires the properties of an ordered concentrated nano-photonic flow, called hyperpolarized. The aim was to investigate the effect of prolonged illumination of an animal with natural daylight passing through a fullerene filter, on behavior, physiological health indices and life expectancy. A long 8-month experiment was conducted on 12 male mice at the age of 1 year. Half of them were constantly under "fullerene" light (experimental group), the rest - under natural light (control). The spectrum of light passing through the fullerene filter was characterized by a weakened short-wavelength range and power density. Weekly we determined the body weight, recorded motor activity (open field test), dynamics of natural behavioral reactions (sleeping, eating, running, washing). It was found that after 9 weeks of observation the body weight in the control group animals began to decrease gradually (one of the signs of aging). At the same time, the average body weight in the animals of the experimental group remained at the same level or even increased. The maximum difference between the two groups occurred 32 weeks after the start of the experiment. The body weight of animals receiving fullerene light was $38.25 \mathrm{~g} \mathrm{(20.5 \%} \mathrm{increase)} \mathrm{in} \mathrm{this} \mathrm{period,} \mathrm{while} \mathrm{in} \mathrm{the} \mathrm{control} \mathrm{group} \mathrm{it} \mathrm{fell} \mathrm{down} \mathrm{to} 27.42 \mathrm{~g}$ (a decrease of $13.8 \%$ ). The test "open field" showed that as the natural aging of animals continues their motor activity decreases. However, in the control group, this process is faster than in animals under the "fullerene" light. After 8 weeks of observation, the mobility of animals in the experimental group was significantly higher than in the control group. It is proved that the "fullerene" light preserves the natural behavior of animals (sleeping, eating behavior, etc.). The animals of the experimental group lived for a total of 1,032 days, and the control animals - 1,036 days. Nevertheless, the quality of life of the "fullerene" animals was the best. This was evidenced by better dynamics of body weight, greater motor activity, and absence of negative behavioral changes. Thus, it was shown for the first time that for a long time up to 8 months illumination of the animal's body with light transmitted through a Nano photonic fullerene filter does not adversely affect physical and functional indices and some of them even improve. Development of some signs of aging is slowing down: weight loss and decreased mobility, although there is no reliable difference in life expectancy between experimental and control animals. The obtained results create the prospect of using fullerene filters for improving ergonomics of the dwelling (fullerene window), everyday use (fullerene glasses), medical purposes (light therapy devices with fullerene filters), for plants (fullerene greenhouses), etc.
\end{abstract}

Key words: Fullerene, hyperpolarization, light, aging, motor activity, body weight, natural behavior of mice, open field test.

\section{Introduction}

Fullerene is a recently discovered new form of carbon. In 1996, Kroto et al. [1] were awarded the Nobel Prize in chemistry for the discovery of fullerenes. It is well known that carbon in its compounds (coal, diamond, graphite) forms structures in the atomic state.

Corresponding author: Sergiy Gulyar, M.D., Ph.D., D.S., Prof., research fields: medicine and physiology.
Fullerene (4th state of carbon) is a molecular structure. The most frequent and studied form of fullerene is the 60 -atom $\left(\mathrm{C}_{60}\right)$. It has a spherical structure with carbon atoms located on its surface, connected in the form of 5- and 6-angles (12 pentagons and 20 hexagons) and with a relative "emptiness" inside. Molecule $\mathrm{C}_{60}$ (truncated icosahedron) is a dynamic vibrational-rotational structure. In hexes, there are holes through which, under certain conditions, small 
atoms or quanta can penetrate inside. $\mathrm{C}_{60}$ is a molecular crystal. Several $\mathrm{C}_{60}$ molecules create a cubic crystal lattice, based on point symmetry. Due to the presence of $60 \pi$-electrons, each $\mathrm{C}_{60}$ molecule has pulling forces that determine the distance between the molecules, with no friction, and the $\mathrm{C}_{60}$ molecule randomly rotates at a rate of about $3 \times 10^{10} \mathrm{~s}^{-1}$.

Today much attention is paid to the problem of using fullerene not only in technology, but also in medicine and pharmacology. It is well known that almost any disease is accompanied by excessive formation in of free radicals the body. Their accumulation generates an avalanche of negative oxidative biological processes. Fullerenes are capable of neutralizing them. Hydroxylated fullerene $\mathrm{C}_{60}(\mathrm{OH}) 26$, which is highly soluble in water, has a significantly higher antioxidant activity than vitamins "E" and "A". Unlike these single-use antioxidants (one molecule of the vitamin neutralizes only one radical particle), fullerenes are reusable: they collect on their surface several radicals that self-destruct. Fullerenes are considered to be an effective trap for free radicals [2]. Due to antioxidant properties, fullerenes hypothetically could influence the aging processes in the body. Thus, it has been shown that consumption of fullerene $\mathrm{C}_{60}$ in rats (a solution in olive oil, per os) leads to a significant slowing down of aging, increasing the lifespan of rats by almost half [3].

Optical properties of fullerene were first considered in the works of D. Koruga [4, 5]. It was revealed and confirmed by physical calculations its possibility to influence light radiation. If the flux of light photons enters the molecular structures of fullerene (for example, with the help of Bioptron device [6, 7]), each photon acquires additional changes in its trajectory. They are determined by interaction of interatomic forces that repel photons from the carbon wall of the molecule and shift their trajectory by the angle described by Faraday with respect to the processes of magnetism [8]. Since the fullerene molecule is also in a state of rotation (in a random direction), the photon speed increases. This further facilitates its flight beyond the molecule with a trajectory that has a rotational configuration. Multiple repetition of this cycle for a photon in subsequent fullerene molecules leads to the creation of their vortex motion (toroids Tesla), which enhances the intensity of the initial light flux (Fig. 1). Distribution of photons inside the flow and on the surface of the application is spiraling and obeys Fibonacci law [4, 5].

Investigation of the influence of BIOPTRON-light passed through the fullerene filter on the initial biophysical state of skin of 32 volunteers showed that such light improves epidermal structure of the skin, increasing the amount of collagen fibers, that is, heals the skin [9]. Earlier in our experiments in mice with an experimentally evoked focus of chemical inflammation, we obtained the evidence of analgesic and sedative effect of polarized BIOPTRON light passed through the fullerene filter [10].

The purpose of this work was to investigate the behavioral responses of animals with their prolonged (8 months) stay under conditions of illumination by natural daylight passing through the fullerene filter. We defined our task as to check whether behavior (motor activity, sleeping, eating) differs in this case and physiological health indices (body weight, lifetime) in animals.

\section{Materials and Methods}

\subsection{Animals}

For the experiment, we selected healthy white male mice at the age of 1 year, which corresponds to the age of man of 60 years. Twelve animals were randomly divided into 2 groups (6 each). Each mouse was placed in a separate cage with free access to food and water. All 12 cells were placed on a shelf, which was located near the window, providing approximately equal illumination to the animals. The temperature in the room was maintained in the range of $22-23{ }^{\circ} \mathrm{C}$. 


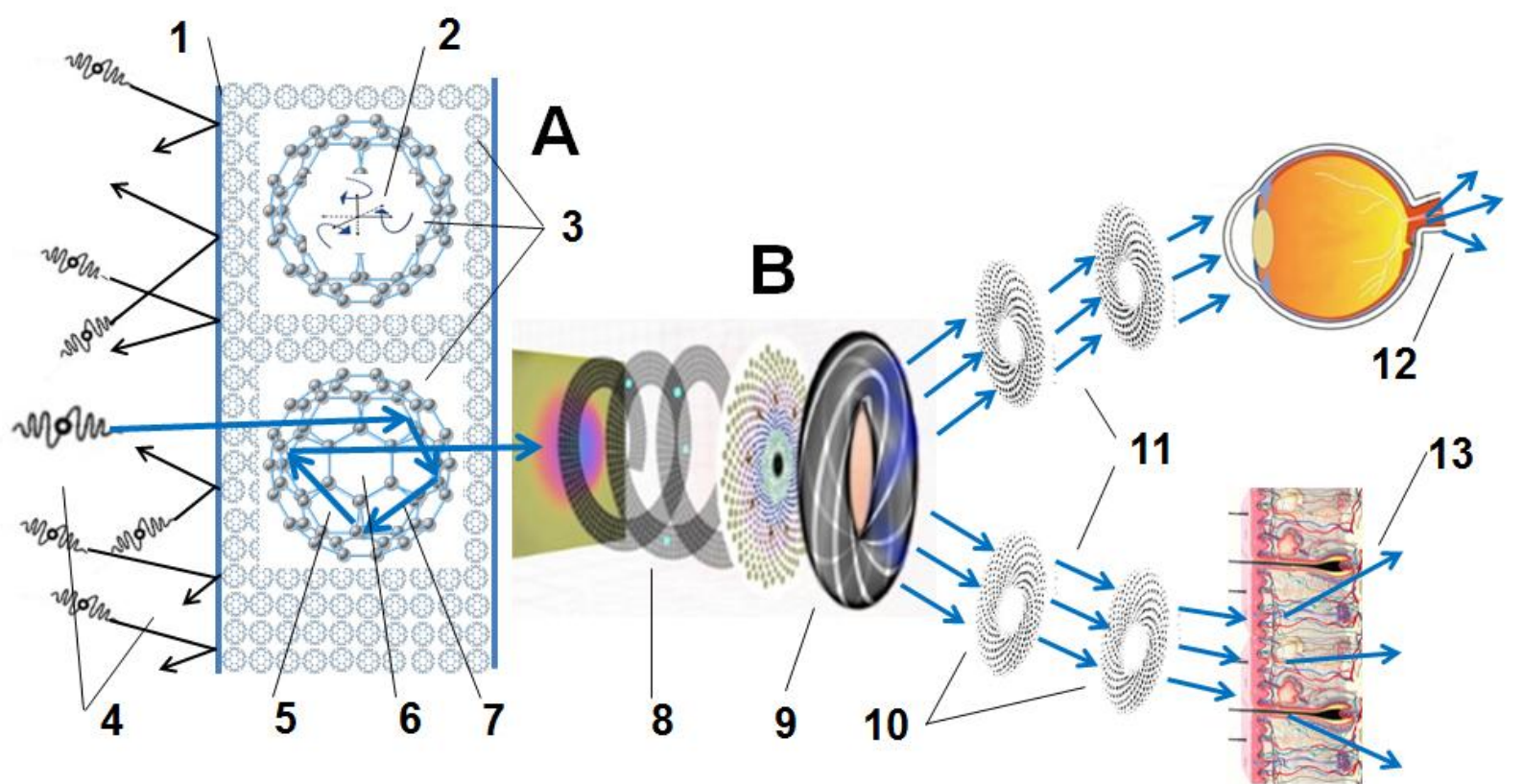

Fig. 1 Structure of the fullerene molecule (A) and change in the direction of light photons, passing through the layer of fullerene molecules $(B)$ :

A: 1-fullerene filter surface; 2-hole, arrows indicate to the direction of rotation; 3 -molecules of fullerene; 4 -photons of scattered (diffuse) light; 5-pentagon; 6-hexagon; 7-trajectory of photon motion inside the fullerene molecule.

B: 8-photon flux formation; 9-Tesla toroid; 10-application zone and distribution of quanta according to Fibonacci scheme; 11-nanophotonic flows; 12 - ocular photon transport pathway; 13 - transdermal pathway of photon transport.

\subsection{Assessment of the Behavior and Physiological State of Animals}

All animals once a week at the same time recorded the following indicators of physical condition and motor activity: body weight, distance traveled in 5 minutes (open field test), duration of behavioral reactions (sleeping, eating, running, washing) for 60 min. We compared the life span of animals that were 8 months under fullerene filters and in the control group (without filters).

After measuring the above parameters in all 12 animals in the initial state, 6 cells with animals were covered with "fullerene" filters (experimental group). The remaining 6 cells did not cover and they formed a control group.

We did animals weighting on electronic scales with accuracy to the second sign, then the data were averaged for each group. The test "open field" determined the distance arbitrarily traversed by each mouse in 5 minutes on a dark smooth fenced surface.
With the help of the video camera placed at the top, all movements of the animal were recorded on the computer. Weekly we also recorded such natural behavioral reactions as sleeping, eating, running, washing. With the help of a special computer program, we determined the duration of the listed behavioral responses in 60 minutes of observation.

\subsection{Statistical Analysis}

For each group, we determined average values of the registered parameters. Data are presented as mean \pm SEM. To determine the statistical significance of the results, we used Student's $t$-test. The difference between the groups was considered statistically significant at $p<0.05$.

\section{Results}

\subsection{Influence of "Fullerene" Light on the Body Weight}

Weekly weighing showed that, before the experiment began, the average body weight in both 
groups was approximately the same (31.75 and $31.8 \mathrm{~g}$ ). It was found that after 9 weeks of observation the body weight in mice of the control group began to gradually decrease (one of the signs of aging). At the same time, the average body weight in the animals of the experimental group remained at the same level or even increased (Fig. 2). Further the difference increased. In the period of 14-25 weeks of observation, the average body weight of animals receiving light through fullerene filters significantly exceeded the control animals (by 3.0-6.6 g). In the following time, this trend persisted. The maximum difference between the two groups manifested in 32 weeks after the start of the experiment. The body weight of animals receiving "fullerene" light was $38.25 \mathrm{~g}$ in this period, whereas in the control group it decreased to $27.42 \mathrm{~g}$. If we estimate these changes in $\%$ from the initial mass, then in the control there was a fall by $13.8 \%$, and in the experiment the increase in mass made up $20.5 \%$.

\subsection{Effect of Fullerene Light on the Mice Motor Activity in "Open Field" Test}

The test "open field" showed that as the natural aging of animals continues their motor activity decreases. However, in the control group, this process is faster than in animals under the "fullerene" light. At the first measurement, the distance traveled in 5 minutes in the control and experimental groups was on average 37.2 and $38.7 \mathrm{~m}$, respectively. At each subsequent testing in both groups there was a decrease in motor activity. Since the 8 th week it stabilized and a difference arose: in the control group, the mobility became lower than in the experimental group (Fig. 3). After 26-33 weeks, the difference between the two groups remained.

In Fig. 4 the trajectories of movement of animals of the control and experimental groups are compared. They illustrate the motor activity, which differs depending on the characteristics of the light exposure.

\subsection{Influence of Fullerene Light on Natural Behavior} (Sleeping, Eating, Running, Washing)

Registration of natural behavioral reactions such as washing (grooming), sleeping, eating and running allows one to judge the spontaneous activity of phylogenetically most stable processes aimed at providing vital activity. In animals, they change under the action of strong stressors, systemic pathology or intoxications. In our case, under the conditions of maximum sparing regime and standardization of

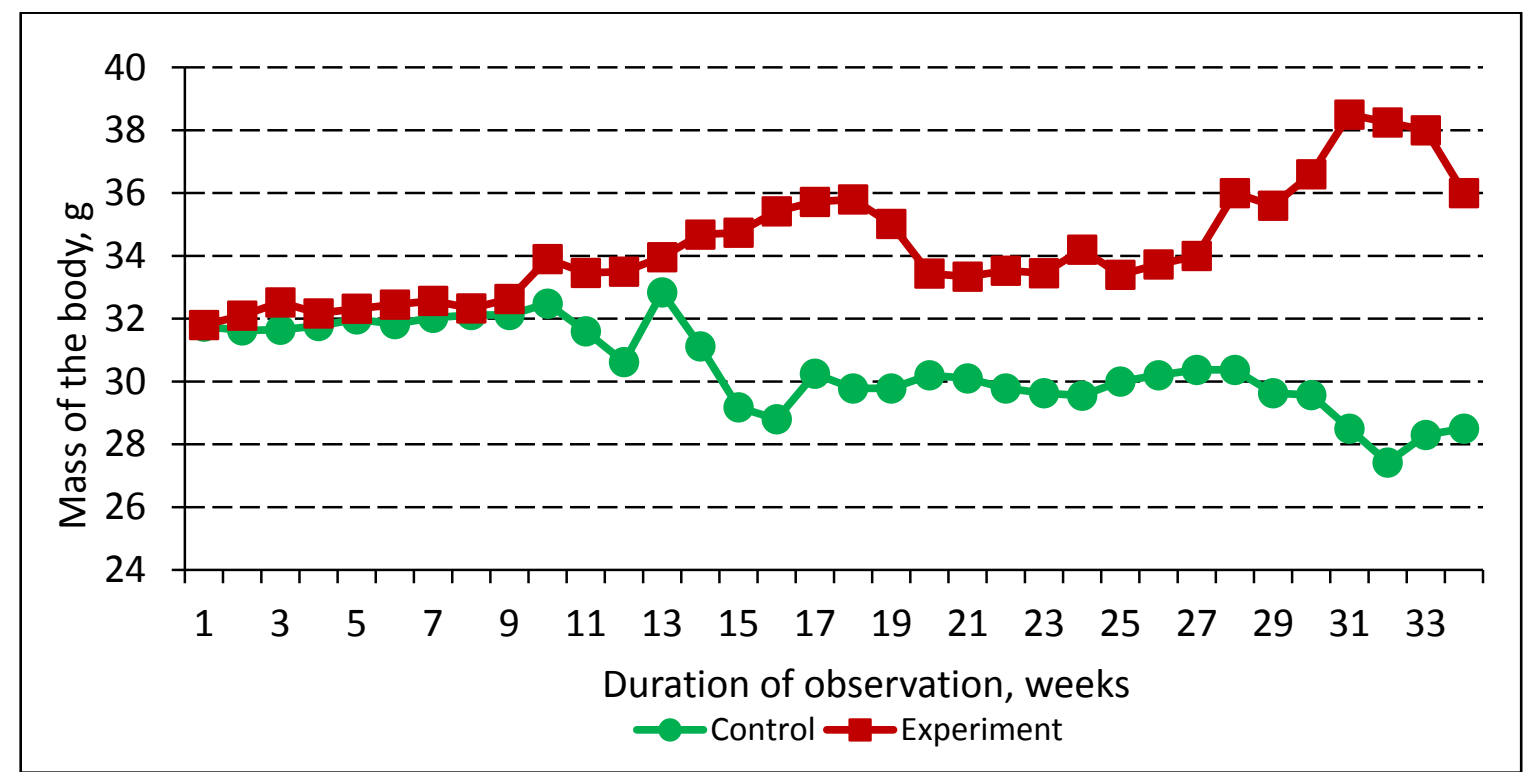

Fig. 2 The body weight changes in the experimental and control groups during 34 weeks of follow-up. Average values for each group. 


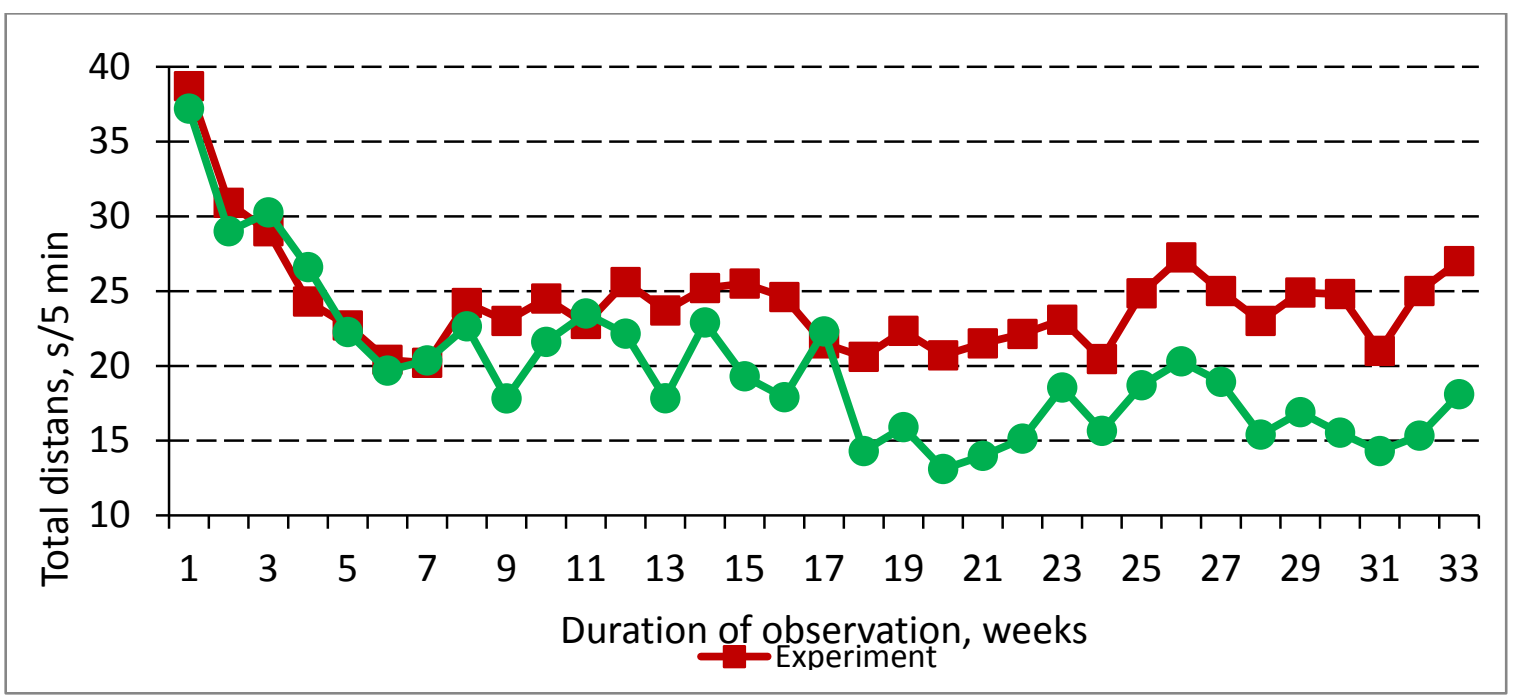

Fig. 3 Average values of the distance traveled in 5 minutes ("open field" test) during 33 weeks of observation in animals under "fullerene illumination" (experiment) and under natural day light (control).
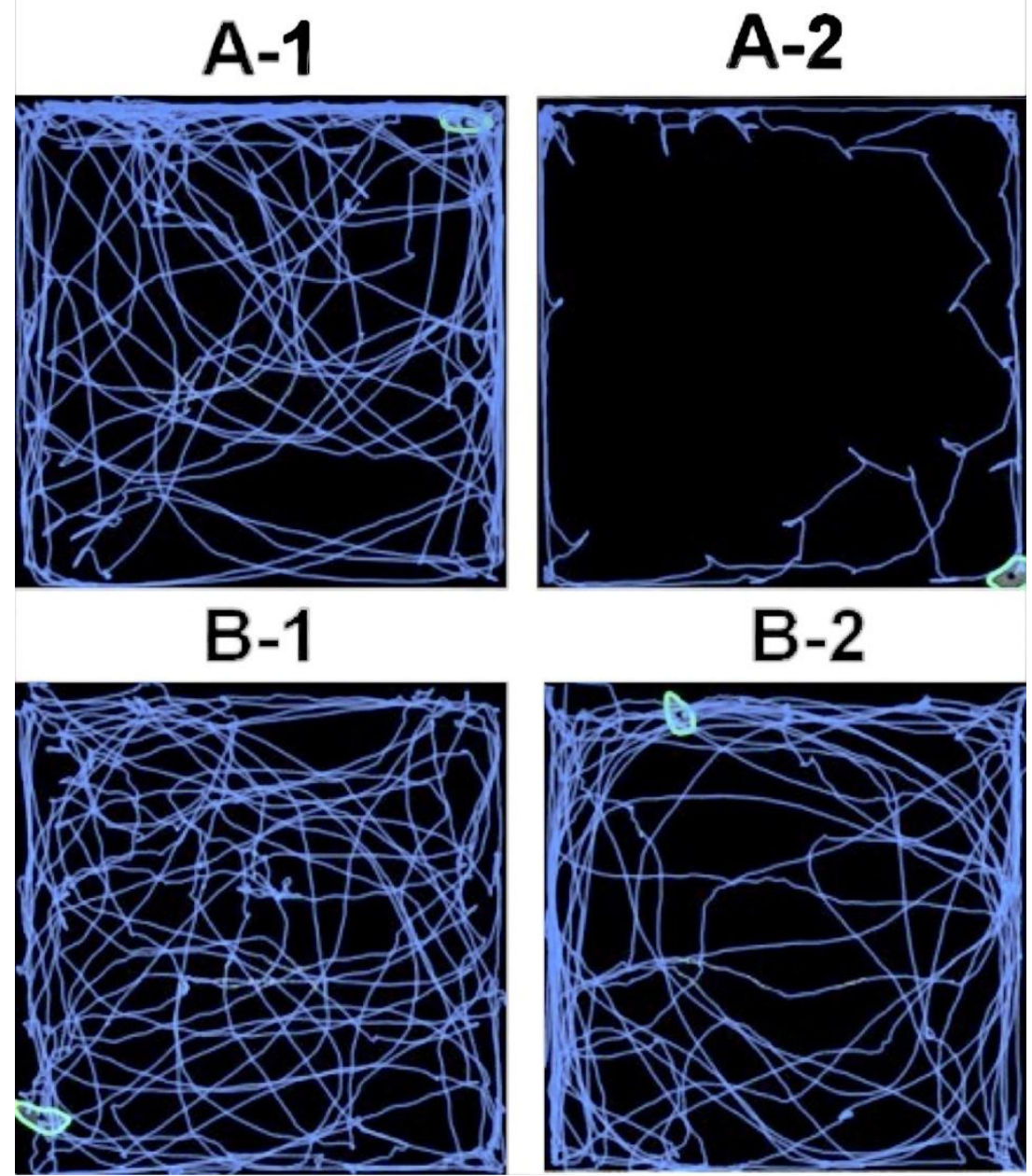

Fig. 4 Representative moving trace of a control (A) and experimental (B) mice in the open field box. Peripheral zone preference (i.e., preference for being in the proximity of the walls) emerges. Moving pattern was registered with Video-tracking system during $300 \mathrm{~s}$.

A-1—1st day: distance 48.9 m; A-2 —after 20 weeks: distance 10.5 m; B-1—1st day: distance 44.9 m; B-2 —after 20 weeks: distance $40.8 \mathrm{~m}$; green color shows the final position of the mouse. 


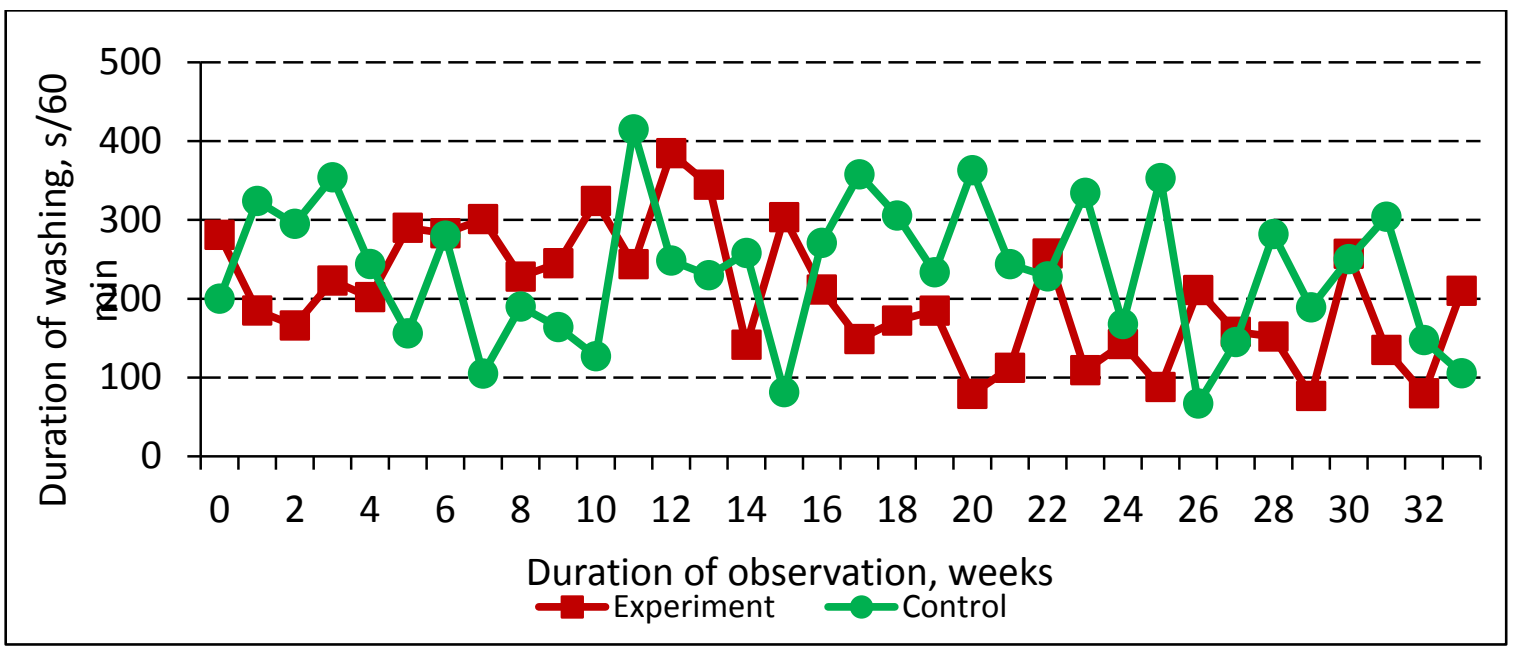

(a)

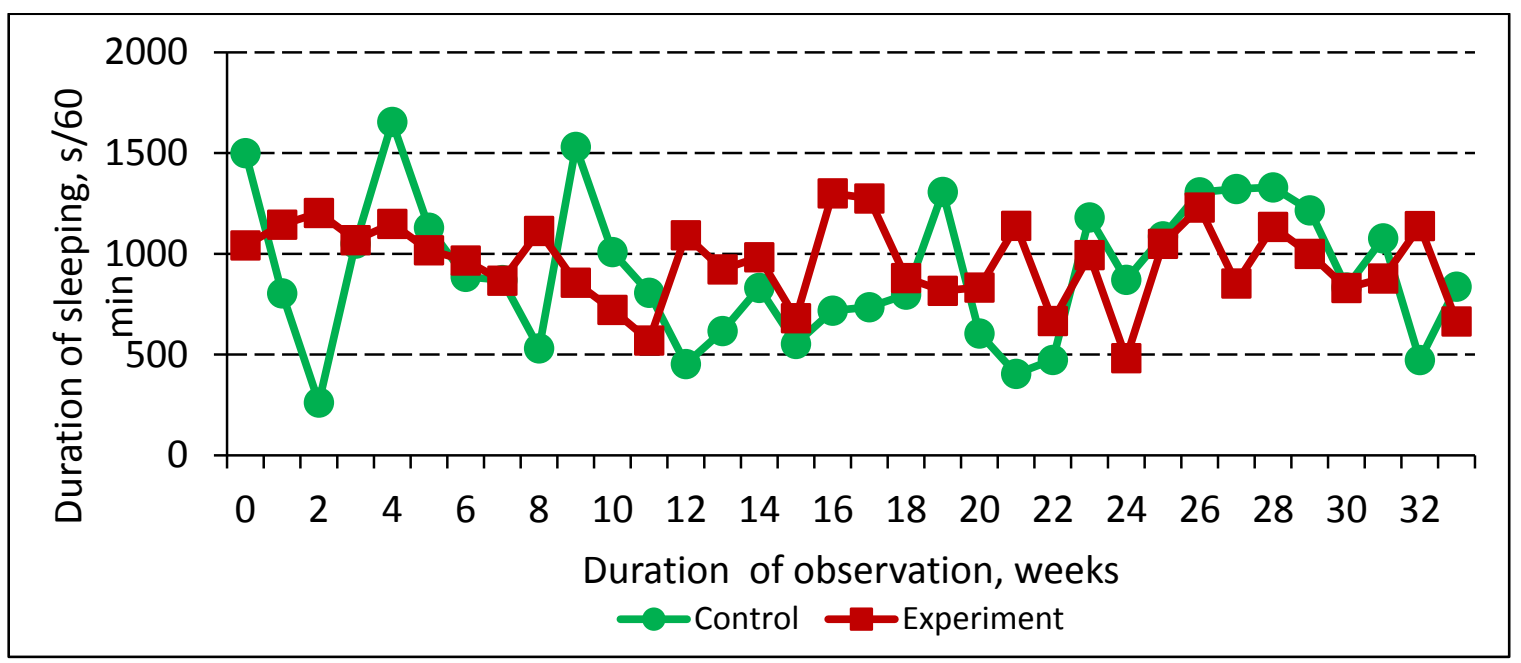

(b)

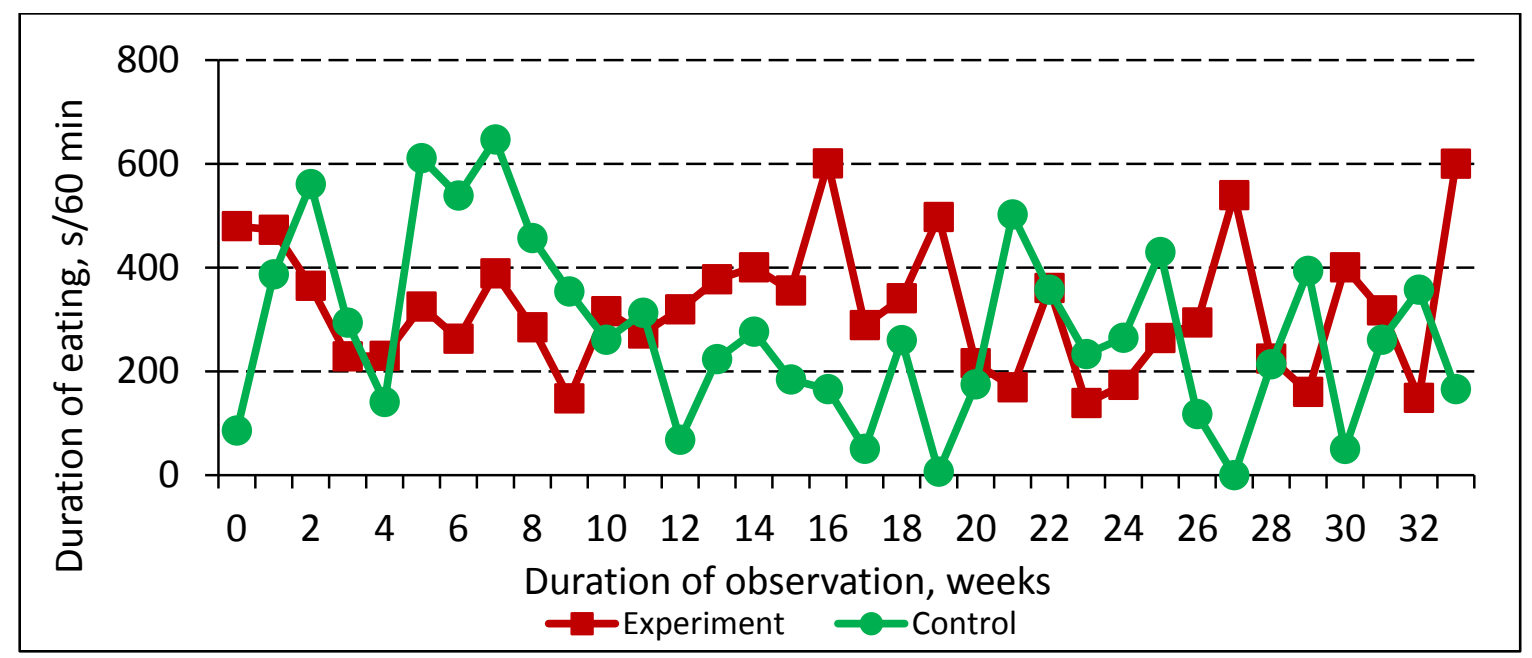

(c) 


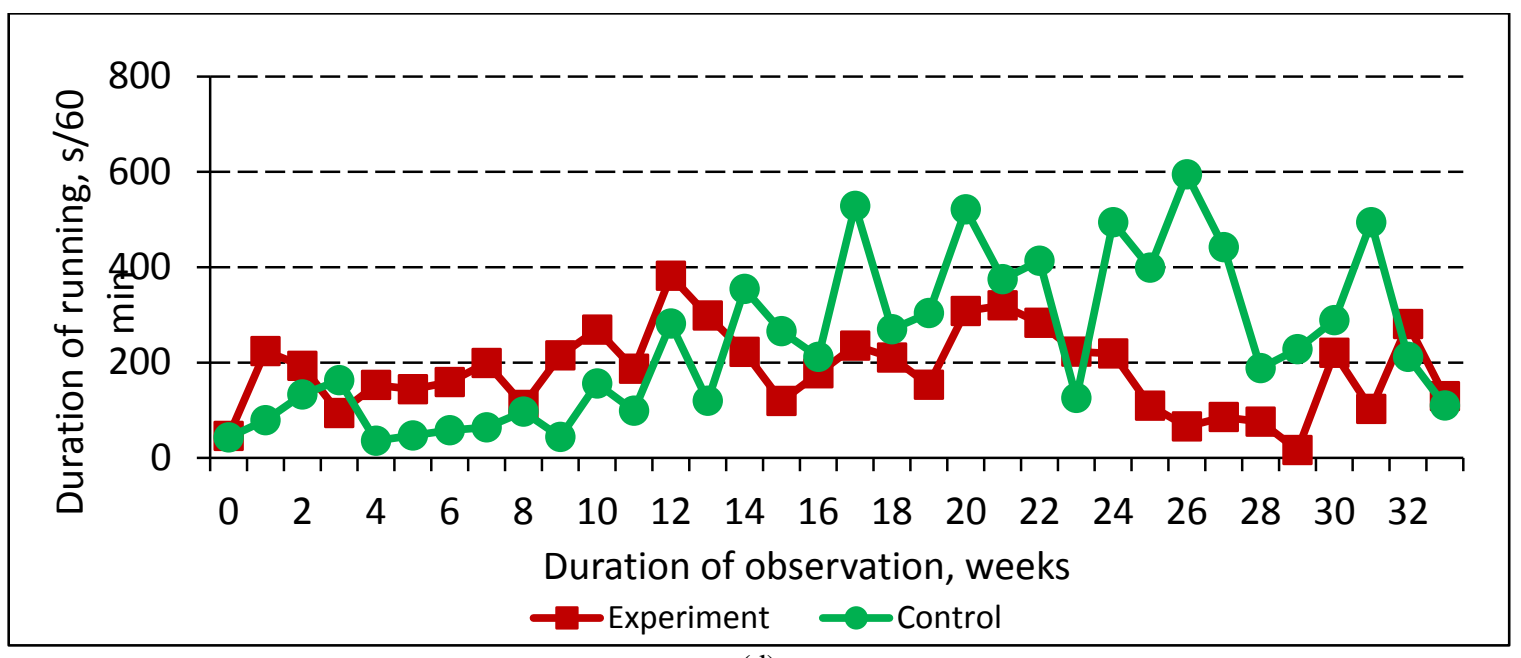

(d)

Fig. 5 Comparison of the duration (for 60 minutes of observation) of behavioral reactions: washing (A), sleeping (B), eating (C), running (D) in animals that were under fullerene illumination (experiment) and under natural daylight (control). There are given average values for each group.

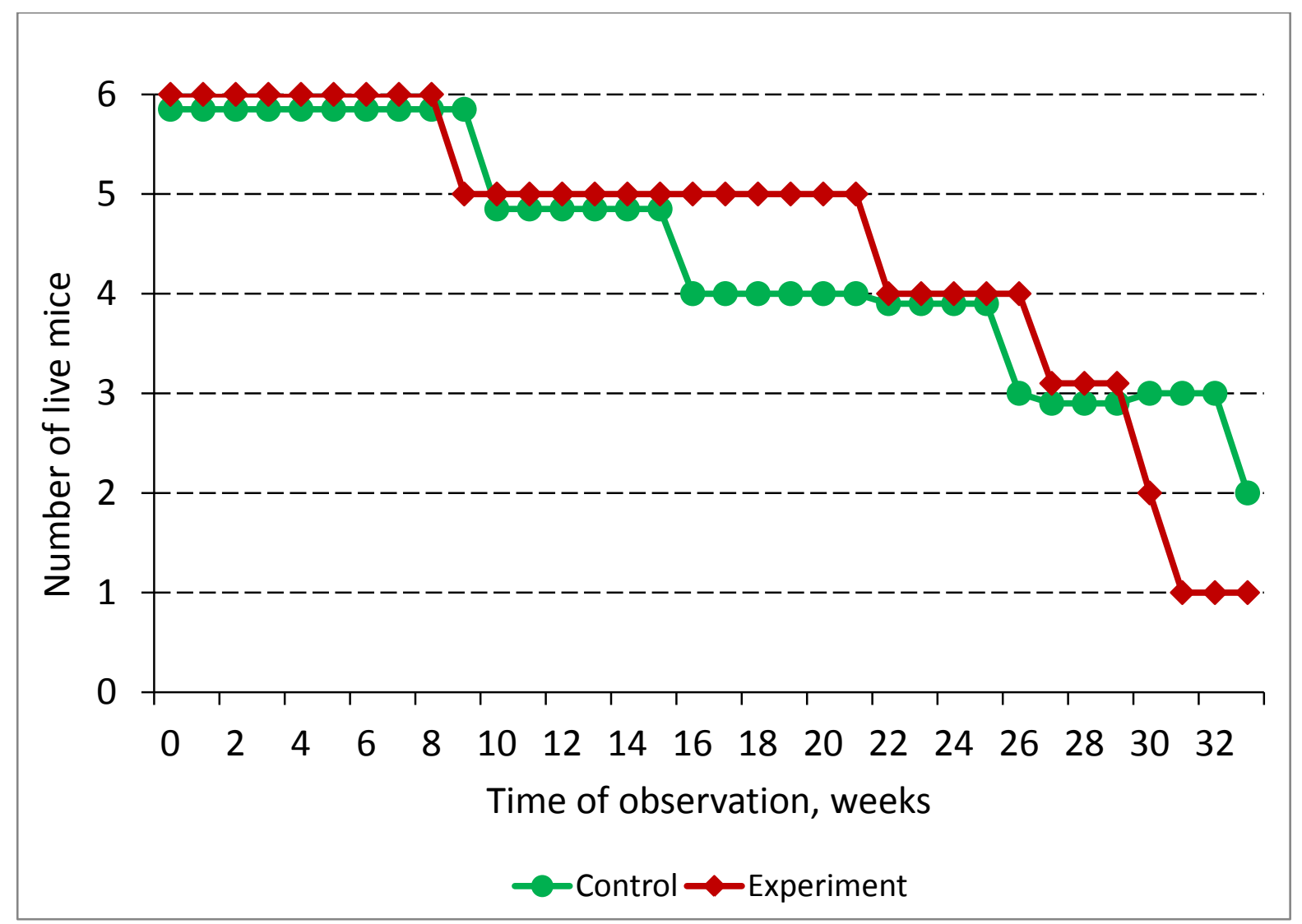

Fig. 6 Survival of animals in the experimental and control groups during 33 weeks of follow-up.

conditions, we did not reveal a significant difference in the behavior of animals that were exposed to "fullerene" light or under similar conditions under natural light. The dynamics of the four behavioral responses in 33 weeks of follow-up are shown in Fig. 5.

3.4 Influence of "Fullerene" Light on the Life Span of Mice 
The results of a comparison of the life span of animals that stayed for 8 months under fullerene filters and the natural daylight are presented as the dynamics of their survival (Fig. 6).

At the beginning of the observation, there were 6 animals in each group (the age of each mouse was 12 months). The first losses occurred in 8-9 weeks (one animal in each group). Then a month and a half later, their number did not change. After 15 weeks of observation in the control group, the number of animals decreased to 4, while in the experimental group there were still 5 animals up to 20 weeks. The number of animals in the two groups (3 individuals in each) became equal in 25-26 weeks. The age of the surviving animals was 20 months, equivalent to about 70 years for man. By the 33rd week of observation, 2 control and 1 experimental mouse remained alive. Because the conditions of keeping all the animals were very good (unlimited access to food and water, stable temperature conditions in the room), we attribute the death of animals to natural aging. Calculation of the total life expectancy of animals showed no significant differences between the two groups. The mice of the experimental group in total during the observation lived 1,032 days, and the control animals-1,036 days.

\section{Discussion}

The present study for the first time experimentally proves that "fullerene" light not only does not adversely affect the physical and functional parameters of animal life, but even improves some of them.

In a prolonged 8-month experiment, biological effects of natural daylight transmitted through a nanophotonic fullerene filter were studied in aging white male lab mice (12 months old) when the entire animal was illuminated. As control, it was a group of animals of the same age that was under natural daylight (without any filters).

The second question that interested us is connected with the gerontological aspect. Our experiments were conducted on aging animals. At the beginning of the study, they were 12 months old, and at the end the age of the surviving mice was 20 months. In the literature there is evidence that fullerene added to food $\left(\mathrm{C}_{60}\right.$ solution in olive oil), increased the lifespan of rats by almost half [3]. Our experimental scheme differed; fullerene was used as a modifier of the light flux. This low-intensity effect, although applied for a long time, did not affect the life expectancy of animals. We did not find an increase in survival rate under the influence of natural daylight, passed through a fullerene filter. This indicator for animals of both groups was approximately the same. The animals of the experimental group lived for a total of 1,032 days, and the control animals-1,036 days. The effect of "fullerene" light on the body surface of an animal is undoubtedly a softer factor than the use of fullerene inside. From our point of view, in the latter case, the factor that influenced animals is sorbing property of carbon, which could reduce the natural intoxication and prolong vital activity of the critical systems of the animal organism.

An important result of this study is detection of the fact that a prolonged (for 8 months) illumination with light passed through the fullerene filter, although it does not prolong life, improves its quality, i.e. indirectly slows down aging in mice. It is known that significant signs of aging are weight loss and decreased mobility. These indicators were significantly better in the group under the "fullerene" light.

Before the start of the experiment, the average body weight in the two groups was approximately the same, and after 9 weeks a distinction became noticable between them. The body weight of mice in the control group began to decrease gradually (one of the signs of aging). At the same time, the average body weight of the animals of the experimental group remained at the same level or even increased. The maximum difference between the two groups occurred in 32 weeks after the start of the experiment. The body weight of animals receiving fullerene light was $38.25 \mathrm{~g}$ (this was $20.5 \%$ increase compared to the beginning of the experiment), 
while in the control group it dropped down to $27.42 \mathrm{~g}$ (a decrease of 13.8\%). It is concluded that prolonged exposure to "fullerene" light contributes to weight gain, which for animals is equivalent to improving their quality of life.

The change in the mobility of animals was evaluated in the "open field" test. The open field method is widely used in the study of behavioral reactions in pharmacology and consists in the study of the motor component of the orienting response and the emotional reactivity of animals. The author of the method is C. S. Hall [11]. Currently, this is one of the most well-known primary behavioral tests for the evaluation of locomotion and research. There are two factors that determine the behavior of rodents in the "open field". One is a positive research drive, originating from the nature of rodents, aimed at exploring new conditions (for finding food and shelter); and the second is a defensive strategy to avoid open and brightly lit spaces (fear of predators). It is known that the behavior of animals in the "open field" depends on genetic factors [12, 13], sex [14], age [15], illumination [16]. In our experiments, only male mice were used. The experiments were conducted at the same time of day, and the same illumination of the field (twilight) was observed. In this regard, the changes observed during 8 months were attributed to the increasing age of mice (from 12 to 20 months).

We evaluated horizontal movements of the mouse (the distance covered in 5 minutes in an "open field"). It was found that the motor activity of animals receiving "fullerene" light was higher than of control animals. At the first measurement, the distance traveled in 5 minutes in the control and experimental groups was approximately equal. In subsequent tests, in both groups there was a decrease in motor activity, which is natural for aging animals. Starting with the 8th week, motor activity stabilizes and a difference arises between the two groups-in the control group, the mobility is lower than in the experimental group.

Since we are considering the long-term effects of a physical factor, another question arises: are there signs of adaptation to it? It is known that there is a phase of the adaptation process, characterized by the phases of acute and sustained adaptation and disadaptation [17]. Based on our data, it can be noted that in the period up to 8 weeks of the experiment, we observed a phase of acute adaptation (Figs. 2 and 3), after which a stable state occurred, which varied for two groups. Natural aging, despite optimal conditions of maintenance, at the end of the experiment, was a factor of adaptation destruction, which became especially noticeable in respect to body weight and mobility. Proceeding from this, it can be assumed that the positive effect associated with the improvement of the quality of life arises after 8 weeks of the systematic application of "fullerene" light. This effect can be useful for a person in the aspect of home ergonomics (fullerene window), everyday use (fullerene glasses), medical purposes (light therapy devices with fullerene filters [10]), for crop production (fullerene greenhouses) and in other areas that will become apparent after receiving experimental confirmation.

\section{Conclusions}

In a long 8-month experiment on elderly white laboratory mice, we studied biological effect of natural daylight passed through the fullerene filter. It has been established that "fullerene" light does not adversely affect physical and functional indices, as well as the natural behavior of animals. This refers to behavioral reactions such as sleeping, eating, running, washing. We revealed the fact of retardation of some signs of aging. Compared to the control group, the body weight of animals increases and activity increases under the action of "fullerene" light. Such dynamics testifies the tendency to improve the quality of life of animals that occurs after 8 weeks of exposure, although it has not affected life expectancy. The results obtained can be a starting point for further research and obtaining evidence on the possibility of using fullerene filters to improve the ergonomics of the dwelling (fullerene 
window), everyday use (fullerene glasses), medical purposes (light therapy devices with fullerene filters), for plants ("fullerene" greenhouses), etc.

\section{Acknowledgments}

This work became possible due to support of Philip Zepter, President of the Zepter International, and Vibor Mulič, Vice President of the Zepter International. Special thanks go to the scientific collaboration of Đuro Koruga, professor of biomedical engineering, and nanotechnology, the University of Belgrade.

\section{References}

[1] Curl, R. F., Jr., Kroto H., and Smalley, R. E. 1996. "The Nobel Prize in Chemistry 1996.” Nobelprize.org.Nobel Media AB 2014. Accessed August 31, 2017. Web: http://www.nobelprize.org/nobel_prizes/chemistry/laureat es/1996/.

[2] Przhiyalgovskaya, N. 2013. "Fullerenes-Sensational Scientific Opening of the 20th Century." Accessed September 1, 2013. http://www.russianscientist.org/files/archive/Nauka/2013 _prziyalgovskaya-29.pdf.

[3] Baati, T., Bourasset, F., Gharbi, N., Njim, L., Abderrabba, M., Kerkeni, A., et al. 2012. "The Prolongation of the Lifespan of Rats by Repeated Oral Administration of [60] Fullerene.” Biomaterials 33 (19): 4936-46.

[4] Koruga, D. 2016. "Nanophotonic Filter Based on $\mathrm{C}_{60}$ for Hyperpolarized Light." Int.and EuropeanPat.App.PCT/EP2016/063174. Applicant: Field point, ZEPTER GROUP filed June 09, 2016 and issued October 28, 2016.

[5] Koruga, D. 2017. "Hyperpolarized Light: Fundamentals of Nano Medical Photonics." In Zepter World Book, Belgrade, 1-306. (in Serbian)

[6] Gulyar, S. A., ed. 2009. Anthology of Light Therapy.Medical BioptronTechnologies. Kyiv: Bogomoletz Institute of Physiology at the National Academy of Sciences of Ukraine, 1-1024. (in Russian)
[7] Gulyar, S. A., and Kosakovskyi, A. L., eds. 2011. Bioptron-PILER-Light Application in Medicine (2nd ed.). Kyiv: Bogomoletz Institute of Physiology at the National Academy of Sciences of Ukraine, 1-256. (in Russian)

[8] Baranova, E. 2010. "Graphene Is Characterized by a Giant Faraday Rotation.” Accessed November 18, 2010.http://sci-lib.com/article912.html. (in Russian)

[9] Morton, J. J. L., Tyryshkin, A. M., Ardavan, A., Benjamin, S. C., Porfyrakis, K. S., Lyon, S. A., et al. 2006. "Bang-Bang Control of Fullerene Qubits Using Ultrafast Phase Gates." Nature Physics 2: 40-3.

[10] Gulyar, S. A., and Tamarova, Z. A. 2017. "Modification of Polychromatic Linear Polarized Light by Nanophotonic Fullerene and Graphene Filter Creates a New Therapeutic Opportunities." Journal of US-China Medical Science 14 (5): 173-91.

[11] Hall, C. S. 1934. "Drive and Emotionality: Factors Associated with Adjustment in the Rat." Journal of Comparative Psychology 17 (1): 89-108.

[12] Sakai, K., and Crochet, S. 2001. "Differentiation of Presumed Serotonergic Dorsal Raphe Neurons in Relation to Behavior and Wake-Sleep States." Neuroscience 104: 1141-55.

[13] Van der Staay, F. J., Kerbusch, S., and Raaijmakers, W. 1990. "Genetic Correlations in Validating Emotionality." Behav Genet 20: 51-62.

[14] Ramos, A., Kangerski, A. L., Basso, P. F., Da Silva Santos, J. E., and Assreuy, J. 2002. "Evaluation of Lewis and SHR Rat Strains as a Genetic Model for the Study of Anxiety and Pain." Behav Brain Res. 129: 113-23.

[15] Colorado, R. A., Shumake, J., Conejo, N. M., Gonzalez-Pardo, H., and Gonzalez-Lima, F. 2006. "Effects of Maternal Separation, Early Handling, and Standard Facility Rearing on Orienting and Impulsive Behavior of Adolescent Rats." Behav Processes 71: 51-8.

[16] Bowman, R. E., Maclusky, N. J., Diaz, S. E., Zrull, M. C., and Luine, V. N. 2006. "Aged Rats: Sex Differences and Responses to Chronic Stress." Brain Res 1126: 156-66.

[17] Gulyar, S. A. 1988. Respiratory Gases Transport during Adaptation of Man to Hyperbaria. Kyiv: Naukova Dumka, 1-296. (in Russian) 